\title{
Physical Activity and Social Interactions of Rural Elementary Children during Recess
}

\author{
Steven L. Prewitt*, Claire Rosselli, Anthony Rosselli \\ Department of Health \& Human Performance, Texas A\&M University - Commerce, Commerce, TX, USA \\ *Corresponding author: steve.prewitt@tamuc.edu \\ Received May 04, 2019; Revised June 29, 2019; Accepted July 25, 2019
}

\begin{abstract}
Recess is an important part of the school day for elementary children. It provides a natural opportunity for children to engage in physical activity, it also allows for young children to develop social skills. There is little research examining physical activity and social interactions among rural elementary children. The purpose of this study was to investigate levels of physical activity, activity type, group size, and social interactions of $3^{\text {rd }}$ and $4^{\text {th }}$ grade students attending a rural elementary school. Students $(\mathrm{N}=67)$ were observed during morning recess $(25$ minutes) using the System for Observing Children's Activity and Relationships during Play (SOCARP). The data were analyzed using multiple independent samples t-tests with Bonferonni adjustments. Initial results showed no significant differences between grade and gender groups in each observation category. Upon further investigation, differences were observed. The results of this study were contradictory and mixed when compared to other studies indicating that other factors such as supervision, equipment, culture and playground markings that may play a significant role in activity levels in children during recess. This study has added to the literature regarding elementary children's physical activity, activity type, group size, and social interactions during recess time. It also adds to the limited literature involving the use of the SOCARP instrument.
\end{abstract}

Keywords: childhood physical activity, systematic observation, social behaviors

Cite This Article: Steven L. Prewitt, Claire Rosselli, and Anthony Rosselli, "Physical Activity and Social Interactions of Rural Elementary Children during Recess." Journal of Physical Activity Research, vol. 4, no. 2 (2019): 114-117. doi: 10.12691/jpar-4-2-6.

\section{Introduction}

Participation in physical activity is a key component for establishing a healthy lifestyle, especially during the elementary grade years. For this age group, recess can provide almost $40 \%$ of a child's daily recommended amount of physical activity. According to the Robert Wood Johnson Foundation [1], recess provides more opportunity for physical activity (42\%) than physical education classes $(32 \%)$ or after-school programs $(26 \%)$. Traditionally, recess time has been viewed as just a time for children to "blow off steam" after periods of sedentary activity. Jarrett [2] has stated that time spent in recess can influence a child's cognitive, social-emotional, and physical development. Pellegrini and Bohn [3] have stated that recess provides the best environment for play, or unstructured activity during the school day and it is this "play" that promotes the physical, social, and cognitive development for children.

For some, there is an alarming decline in the opportunity and duration of recess [4]. Some have cited that issues such as bullying and other antisocial behaviors have led to a reduction or elimination of recess [5]. Others have stated that these incidents are relatively rare [6] and suggest methods for reducing these incidences. The majority of the recent literature has focused primarily on the physical activity occurring with few studies examining activities during recess and how children are interacting socially during this time. Several studies have looked at the role social interactions has on an individual's physical activity $[7,8]$, yet the majority of the research has taken place internationally with few studies examining students in the United States [9,10].

Examining physical activity levels in children is critical; however, another segment to the importance of recess is navigating social constructs. Ridgers, Stratton, and McKenzie [8] examined social interactions during recess and have classified them as either prosocial or antisocial. The authors further suggested that social interactions consisted of verbal, nonverbal, physical, and nonphysical. Coolkens and others [11] found that girls engaged in more prosocial behavior (holding hands, helping each other, and retrieving equipment for others) during recess, whereas boys engaged in more antisocial behavior (pushing, taking equipment, and hitting). Overall these studies suggest that recess provides children more positive social interactions than negative. As children are physically active during recess they are also socially active. The central nature of recess points to not just the physical development of children, but the development of social interactions. It can be suggested that social interactions among peers plays a role in the amount and type of physical activity 
participation. Sevil, Garcia-Gonzalez, Abos, Lanaspa, and Solana state, "peers have been closely linked with physical activity involvement during adolescence" ([12], p.15). The social construct of recess and the impactful nature of peer influence, justifies recess on the grounds for optimal PA and social influence in children.

Finally, research has discussed one more essential factor to successful PA during recess. Sevil et al. [12] highlighted in their findings that school-based motivational interventions increased PA levels. This knowledge concerning intervention paints a picture of exactly how to improve PA levels among children. These concepts suggest that the PA on the playground is of developmental importance and teacher interaction and motivation plays a critical role.

Recess is considered to be the optimal environment for play during the elementary years. As mentioned previously, few studies have examined other variables that can affect physical activity during recess. Furthermore, the majority of research examining these variables have been conducted at urban schools $[9,10]$ as well as schools outside of the United States [7,8,13]. Therefore, the purpose of this study was to examine the physical activity, activity type, group size and social interactions of third and fourth grade rural elementary students during recess. It is hypothesized that the findings from this student will reflect current literature.

\section{Methods}

\subsection{Participants}

Students $(\mathrm{N}=67)$ enrolled in third $(n=32)$ and fourth grade attending a rural public elementary school outside of a major metropolitan city in the Southern region of the United States were recruited for this study. One hundred and sixty students were eligible to participate with 67 (males=33) students $(42 \%)$ actually being observed for this study. Upon obtaining University Institutional Review Board and school administration approval, parental consent and student assent were obtained. The school, classified as a Title I (economically disadvantaged), had an overall student body of 359 students (PreK- $4^{\text {th }}$ grade) with 54.1\% White, 38.8\% Hispanic, and 3.8\% African-American. Of these students, $22.8 \%$ were English Language Learners and $47.5 \%$ were classified as At-Risk.

The school offered two recess sessions per day, morning and lunch time, each lasting approximately 25 minutes. School policy was that each grade level would have their own recess time, eliminating multi-grade interactions. The central play area was recently re-designed with a multi-level covered play structure (six months prior). Other play options included a large grass field with two soccer goals, a paved surface with two fixed basketball goals, and two areas with smaller fixed play structures. Recess times were supervised by grade level teachers. It was noted during the earlier recess $\left(3^{\text {rd }}\right.$ grade), very few pieces of equipment were brought out by the students. Fourth grade recess equipment was generally facilitated by a supervising teacher who would bring out a set number of pieces including playground balls, soccer balls, and mats for kickball/baseball type games. Other equipment consisted of students' personal pieces (i.e. footballs).

\subsection{Protocol}

Data were collected using the System for Observing Children's Activity and Relationships during Play (SOCARP), an assessment tool found to be both reliable and valid when measuring physical activity and social interactions of younger children [8]. SOCARP utilizes a 10 -second observation interval, 10-second recording interval technique to measure physical activity levels, type of activity engaged in, social group size, and various social interactions of a single child. The observation period per subject is 10 minutes. During this particular study, three observers were stationed on the playground before recess began. Observers were randomly assigned two students (one male, one female) to observe during each recess period. A one-minute preparation period was utilized allowing the observed student time to engage in recess (i.e. waiting for friends, running to play area, etc.). Once data collection began, observers were cued to the 10 -second observation/10-second recording intervals via audio cues from their cellular phones.

The SOCARP instrument was chosen as it gives more detail of what is occurring during recess compared to other tools that record physical activity levels only. SOCARP does assess physical activity levels (lying, sitting, standing, walking, very active) as well as activity type (sports, games, locomotion, sedentary), social group size (alone, small 2-4, medium 4-9, large 10+) and social interactions (none, physical sportsmanship, verbal sportsmanship, physical conflict, verbal conflict, ignore antisocial behavior) [8].

\subsection{Data Analysis}

To assess the percentage of time each child spent in the four different SOCARP categories, descriptive statistics were run. Independent samples t-tests were used to examine whether statistically significant differences existed between grade levels, gender, and equipment or no equipment of the SOCARP factors. Bonferroni adjustment was used to accommodate for the multiple t-tests calculated (i.e., activity level and social interaction had six independent t-tests calculated, so the adjusted $p$-value was $<.008$; group size and activity type had four independent $\mathrm{t}$ tests run, so the adjusted $p$-value was <.0125). To determine the correct $t$ and $p$ values, Levene's test for equality of variances was used. Finally, Cohen's $d$ statistic was calculated to report effect size differences between the groups.

\section{Results}

The findings from the independent samples t-tests are reported below. Each category will be addressed separately.

\subsection{Activity Level}

Overall, participants engaged in moderate-to-vigorous intensity physical activity during recess. However, no 
significant differences were found in activity levels between grades [Wilks' $\lambda=.84, F(6,60)=1.88, p=.10$ ], gender [Wilks' $\lambda=.90, F(6,60)=1.06, p=.40$ ], or gender/grade interactions [Wilks' $\lambda=.75, F(18,164.53)$ $=.96, p=.51]$. No significant differences were observed when accounting for the presence of equipment [Wilks' $\lambda=.84, F(6,60)=1.92, p=.09]$.

\subsection{Activity Type}

No statistically significant differences were observed in activity type and gender [Wilks' $\lambda=.89, F(4,62)=1.97$, $p=.11]$. There were significant differences in grade level and activity type [Wilks' $\lambda=.79, F(4,62)=4.05$, $p=.006]$. Fourth graders spent more time playing sports than $3^{\text {rd }}$ graders $(t=-3.85, p<.001, d=.92)$. Significant differences were also observed within gender and grade level [Wilks' $\lambda=.64, F(12,159)=2.43, p=.006$ ]. Fourth grade females were observed to have spent significantly more time playing sports than $3^{\text {rd }}$ grade males $(t=-4.01$, $p=.001, d=1.34)$ and $3^{\text {rd }}$ grade females $(t=-3.86$, $p=.001, d=1.29)$. Finally, the presence of equipment significantly affected the type of activity [Wilks' $\lambda=.83$, $F(4,62)=3.27, p=.02]$. When equipment was present, sports were played more than when no equipment was present $(t=-3.21, p=.003, d=.83)$.

\subsection{Group Size}

No significant differences in group size and gender were observed [Wilks' $\lambda=.90, F(4,62)=1.72, p=.16$ ] Grade levels did differ significantly in group size with $3^{\text {rd }}$ graders spending more time in small groups than $4^{\text {th }}$ graders $(t=3.27, p=.002, d=.79)$ and $4^{\text {th }}$ graders spending more time in large groups than $3^{\text {rd }}$ graders $(t=-3.21, p=.003, d=.77)$. Investigating these differences showed that $3^{\text {rd }}$ grade males $(t=4.34, p<.001$, $d=1.51)$ and $3^{\text {rd }}$ grade females $(t=3.29, p=.002$, $d=1.12$ ) spent more time in small groups than $4^{\text {th }}$ grade females. Furthermore, $3^{\text {rd }}$ grade males spent less time in large groups than $4^{\text {th }}$ grade females $(t=-3.14, p=.005$, $d=1.05)$. As was reported in the previous section, the presence of sports equipment also showed significant differences; those with equipment spent more time in large groups than those without equipment $(t=03.12, p=.004$, $d=.81)$.

\subsection{Social Interaction}

Overall, participants demonstrated positive social interactions. However, no significant differences were observed between gender [Wilks' $\lambda=.86, F(6,60)=1.63$, $p=.16$ ], grade level [Wilks' $\lambda=.93, F(6,60)=.79$, $p=.58$ ], gender/grade level [Wilks' $\lambda=.78, F(18,165)$ $=.83, p=.66]$, or the presence of equipment [Wilks' $\lambda=.89, F(6,60)=1.19, p=.33]$ and social interaction.

\section{Discussion}

The purpose of this study was to investigate physical activity levels, types of activity, group size, and social interactions of $3^{\text {rd }}$ and $4^{\text {th }}$ grade students at a rural elementary school, using the SOCARP instrument. The results from this study suggest that were no significant differences between males and females for the four areas. These findings are contrary to previous studies [10,13], especially in regards to physical activity levels. However, others have reported that girls can be just as active as their male classmates during more structured physical activity time [14].

Other findings of interest include differences in group size when comparing $3^{\text {rd }}$ and $4^{\text {th }}$ grade boys. The $3^{\text {rd }}$ grade group preferred to spend more time in small (2-4 individuals) than the $4^{\text {th }}$ graders, who preferred to engage in large groups (10+ individuals), which could be explained by the preferred activity type. The older boys were engaged in more organized sport activities rather than unstructured playground-type activities. Another interesting discovery was the higher percentage of time that the $4^{\text {th }}$ grade girls spent engaged in sport activities (45\%) compared to the other groups. This could be attributed to having a teacher-led game during recess.

Lastly, all of the groups reported very high percentage $(52 \%)$ of positive social interactions (physical and verbal) and very low percentage $(1.75 \%)$ of negative interactions. Since this was an observational study, there was no information as to whether this was typical or due to a campus-wide leadership program.

Recess is an important time for students to engage in physical activity. It also provides opportunities for social development, especially during the elementary grades. In this study, students exhibited appropriate levels of MVI and high levels of positive social interactions. There were two main limitations observed with this particular study. First would be the timing of the observations, October and November. Several times during the study, students were not allowed outside recess due to inclement weather, which led to three observation sessions being cancelled. Secondly, the SOCARP instrument is a relatively newer observation tool, and has not been utilized very often in the United States. As with most new inventories, the amount of training literature is limited. While the lead investigator was familiar with the tool, others were not which could lead to potential inter-rater unreliability. As SOCARP has shown to be a useful tool in the evaluation of physical activity and social interactions during recess time, the development of training material for future use might be helpful.

With an increase in the concern regarding children's physical activity, especially during recess, it is important to examine not only the physical aspect of recess, but also to explore group dynamics during these times. Results and information from this study will allow other researchers to investigate and design further interventions that will help to promote safe, fun, and physically active recess opportunities.

\section{References}

[1] Robert Wood Johnson Foundation. (2007). Recess Rules: Why the undervalued playtime may be America's best investment for healthy kids and healthy schools. Available from http://www.rwjf.org/vulnerablepopulations/product.jsp?id=20591.

[2] Jarrett, O.S. (2002). Recess in elementary school: What does the research say? Retrieved October 14, 2017, from www.eric.ed.gov. 
[3] Pellegrini A.D. \& Bohn C.M. (2005). The role of recess in children's cognitive performance and school adjustment. Education Research 34(1), 13-19.

[4] Jarrett O.S. (2013). A research-based case for recess. Retrieved October 12, 2017 from www.usplaycoalition.clemson.edu

[5] Jarrett, O., \& Waite-Stupiansky S. (2009). Recess - it's indispensable! Young Child 64(5), 66-69.

[6] Pellegrini A.D. (2008). The recess debate: A disjuncture between educational policy and scientific research. American Journal of Play, 1(2), 181-191.

[7] Ridgers, N.D., Fairclough, S.J., \& Stratton, G. (2010). Variables associated with children's physical activity levels during recess: the A-CLASS project. International Journal of Behavioral Nutrition and Physical Activity, 7(74), 1-8.

[8] Ridgers, N.D., Stratton, G., \& McKenzie, T.L. (2010). Reliability and validity of the system for observing children's activity and relationships during play (SOCARP). Journal of Physical Activity \& Health, 7, 17-25.

[9] Woods, A.M., Graber, K., \& Daum, D. (2012). Children's recess physical activity: movement patterns and preferences. Journal of Teaching Physical Education, 31, 146-162.
[10] Woods, A.M., Graber, K.C., Daum, D.N., \& Gentry, C. (2015). Young school children's recess physical activity: movement patterns and preferences. Journal of Teaching Physical Education, 34, 496-516.

[11] Coolkens, R., Ward, P., Seghers, J., \& Iserbyt, P. (2018). The effect of organized versus supervised recess on elementary school children's participation, physical activity, play, and social behavior: A cluster randomized controlled trial. Journal of Physical Activity \& Health, 15(10), 747-754.

[12] Sevil, J., Garcia-Gonzalez, L., Abos, A., Lanaspa, E. G., \& Solana, A. A. (2010). Which school community agents influence adolescents' motivational outcomes and physical activity? Are more autonomy-supportive relationships necessarily better? International Journal of Environmental Research and Public Health, 15, 1-21.

[13] Ridgers, N.D., Carter, L.M., Stratton, G., \& McKenzie, T.L. (2011). Examining children's physical activity and play behaviors during school playtime over time. Health Education Research, 24(4), 586-595.

[14] Sarkin, J.A., McKenzie, T.L., \& Sallis, J.F. (1997). Gender differences in physical activity during fifth-grade physical education and recess periods. Journal of Teaching Physical Education, 17, 99-106.

(C) The Author(s) 2019. This article is an open access article distributed under the terms and conditions of the Creative Commons Attribution (CC BY) license (http://creativecommons.org/licenses/by/4.0/) 